\title{
Conical Dispersion and Effective Zero Refractive Index in Photonic Quasicrystals
}

\author{
Jian-Wen Dong, ${ }^{1,}$ Ming-Li Chang, ${ }^{1}$ Xue-Qin Huang, ${ }^{2}$ Zhi Hong Hang, ${ }^{3}$ Zhi-Chao Zhong, ${ }^{1}$ Wen-Jie Chen, ${ }^{1}$ \\ Zhan-Yun Huang, ${ }^{1}$ and C. T. Chan ${ }^{2}$ \\ ${ }^{1}$ School of Physics and Engineering and State Key Laboratory of Optoelectronic Materials and Technologies, \\ Sun Yat-Sen University, Guangzhou 510275, China \\ ${ }^{2}$ Department of Physics and the Institute for Advanced Study, The Hong Kong University of Science and Technology, Hong Kong, China \\ ${ }^{3}$ Department of Physics, Soochow University, 1 Shizi Street, Suzhou 215006, China
}

(Received 17 October 2014; published 20 April 2015)

\begin{abstract}
It is recognized that for a certain class of periodic photonic crystals, conical dispersion can be related to a zero-refractive index. It is not obvious whether such a notion can be extended to a noncrystalline system. We show that certain photonic quasicrystalline approximants have conical dispersions at the zone center with a triply degenerate state at the Dirac frequency, which is the necessary condition to qualify as a zerorefractive-index medium. The states in the conical dispersions are extended and have a nearly constant phase. Experimental characterizations of finite-sized samples show evidence that the photonic quasicrystals do behave as a near zero-refractive-index material around the Dirac frequency.
\end{abstract}

DOI: 10.1103/PhysRevLett.114.163901

PACS numbers: 42.70.Qs, 41.20.Jb, 61.44.Br

Photonic crystals and metamaterials are artificial structures that have attracted much attention because of their ability to control wave propagation. Recently, a class of periodic dielectric photonic crystals [1-3] with conical dispersions at $\mathbf{k}=\mathbf{0}$ [4-6] have been demonstrated to behave as a zero-refractive-index material at Dirac frequency. Such dielectric photonic crystals [1-10] have the advantage of being nearly lossless as no metal resonators are needed, and have a finite group velocity while the phase velocity is infinite [2]. These designs have been realized and characterized from microwave [1] to optical frequencies [10]. However, the connection between conical dispersions at $\mathbf{k}=\mathbf{0}$ and zero-refractive index were built upon periodicity. The conical dispersion was obtained by tuning the system parameters of "one-atom-per-unit-cell" photonic crystals with a well-defined photonic band structure. Whether a conical dispersion can exist in a nonperiodic system is still an interesting and open question. Furthermore, the claim that a system behaves like a zero-index medium implicitly assumes that an effective medium description could be applied. While not explicitly stated, many coherent-potential-approximation-type effective medium theories, employed to map a Dirac cone to zero index [11], assume that each scatter resides in the same environment. Although this assumption of periodicity is not needed in the $\omega \rightarrow 0, k \rightarrow 0$ limit, it is not immediately obvious that such effective medium description can be applied to nonperiodic systems if we consider effective parameters at $k \rightarrow 0$ but at a finite frequency such as a Dirac point. Can a nonperiodic system behave operationally as if it has zero-refractive index?

Photonic quasicrystal (PQC) is constructed by building blocks positioned on well-designed patterns but lacks translational symmetry [12-23]. Nonetheless, PQC can still have relatively sharp diffraction patterns due to longrange order. Such patterns confirm the existence of wave scattering and interference, providing similar functionalities as periodic counterparts, such as photonic band gaps [12-15], negative refraction [16], lasing [17-19], and nonlinear light propagations [20-22]. We will show theoretically and experimentally that some two-dimensional photonic quasicrystalline approximants can possess conical dispersion at $\mathbf{k}=\mathbf{0}$, and their finite-sized counterparts can behave like a zero-refractive-index medium as evidenced by different experimental measurements.

In this Letter, we show the existence of conical dispersions and extended states with zero-refractive-index characteristics in some PQCs, and experimentally characterize these states. The extended states are close to the Dirac frequency, and form two cones intersecting at a "Dirac point." The eigenmodes have almost constant field intensity at each quasicrystalline site, regardless of the size and the boundary of the structure. This is in contrast to most other modes that show highly uneven field distributions typically found in localized modes. Furthermore, near-zero phase change and the channel cloaking effect, the signatures of a zero-refractive-index medium, are experimentally confirmed in 12-fold PQCs. As another experimental demonstration of an effective zero index, a prism-shaped morphology has been employed to achieve asymmetric transmission inside a multichannel waveguide.

Consider a transverse magnetic polarized plane wave propagating in a two dimensional (2D) 12 -fold $\mathrm{PQC}$, which is constructed by placing dielectric rods at the 2D dodecagonal lattice sites generated by the square-triangle tiling model [24]. As the PQC is aperiodic and thus the eigenmodes cannot be presented as a band structure, it is still possible to study the photonic dispersion by calculating 
photonic bands using so-called "crystalline approximants," which are periodic systems with supercells containing quasicrystalline fragments. Implemented in the 12-fold PQC, two kinds of hexagonal supercells possessing $C_{6 v}$ symmetry are selected, as shown in Figs. 1(a) and 1(c). The smaller and bigger supercells, respectively, are named "first level" and "second level" crystalline approximants and are assigned lattice constants of $(2+\sqrt{3}) a$ and $(2+\sqrt{3})^{2} a$, where $a$ is the distance between two nearest neighboring rods. The radius of the rod is $0.1929 a$ for the first level cell and $0.1923 a$ for the second level. The dielectric constant of the $\operatorname{rod}$ is $\varepsilon=12.75$. Note that each crystalline approximant is slightly different from a "true" quasicrystal in its own way; therefore, the rod radii needed to realize conical dispersion is likewise slightly different. As shown in Figs. 1(b) and 1(d) with three-dimensional band structures near $\mathbf{k}=\mathbf{0}$, the salient features are very similar. Both have two branches with conical dispersions (purple) touching at one point and a flat orange branch meeting the singular point of the cones at $\mathbf{k}=\mathbf{0}$ at the "Dirac frequency" of $0.5584 c / a$ and $0.5595 c / a$, for the respective supercells.

Here, $c$ is the speed of light.

We emphasize that the conical dispersion originates from the intersecting branches of dispersion without level repulsion, and such linear crossings are protected by symmetry. And while quasicrystals possess high enough rotational symmetry to "protect" conical dispersions, such a triply degenerate state is not generic for arbitrary structural parameters, therefore, the radius of the rods needs to be tuned to achieve the conical dispersions for a given value of the dielectric constant. Alternatively, one can fix the radius
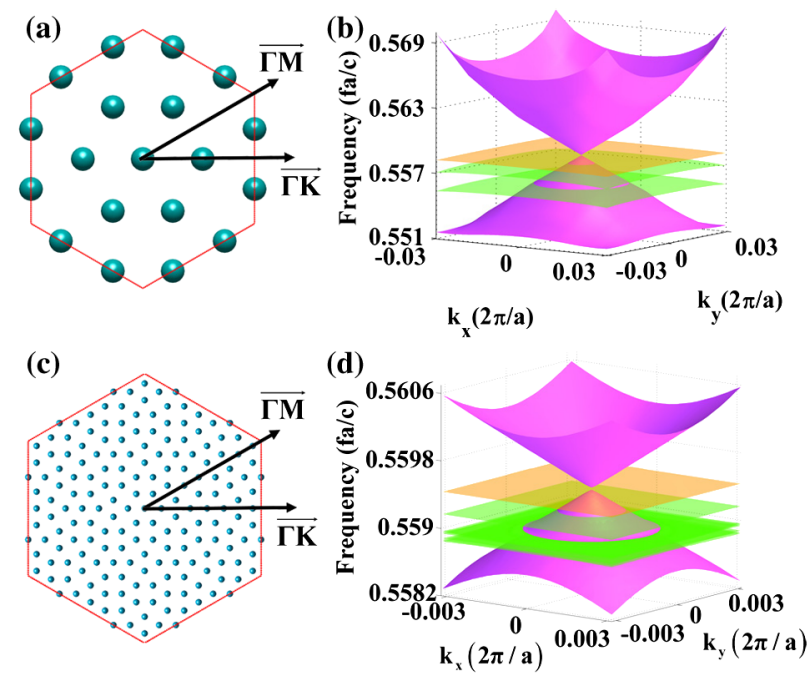

FIG. 1 (color online). Supercell crystalline approximants and conical band dispersion near $\mathbf{k}=\mathbf{0}$ for 12 -fold photonic quasicrystals. (a) and (b) are for the first level, while (c) and (d) are for the second level. Conical dispersions (purple) with a Dirac point are evident, with a flat band (orange) intersecting the Dirac frequency. The lower cone is intersected by multiple flat sheets (green) of localized ring modes. and adjust the dielectric constant. In addition, detailed analysis shows that the conical dispersions in Fig. 1 are generated by monopolar and dipolar modes (Supplemental Material [25], Appendix A), satisfying the necessary condition to achieve zero-refractive-index behavior (to be discussed later). The similarity in structural parameters and the Dirac frequency of both supercells implies that the photonic quasicrystalline approximants of different sizes with conical dispersions may share the same physics. Such physical properties should be a characteristic of the quasicrystalline arrangement rather than the size of the artificial supercell, considering that as the size of the crystalline approximant supercell increases, the conical dispersions (which provide extended modes) and a longitudinal flat band remain touching at the Dirac frequency. In addition, flat bands below the Dirac point [green sheets in Figs. 1(b) and 1(d)] are found to be localized ring modes [Fig. S1(d)]. Increasingly more dispersionless ring modes appear within a particular frequency window as the size of the supercell increases. Furthermore, conical dispersions induced by the triply accidental degenerated state are observed in eightfold symmetry photonic quasicrystalline approximants (Supplemental Material [25], Appendix B). In short, the dispersion of the $\mathrm{PQC}$ near $\mathbf{k}=\mathbf{0}$ comprised two cones touching at the Dirac point, an additional flat band passing through the Dirac point and many additional dispersionless "ring modes." This is the necessary condition to be qualified as a zero-refraction-index medium. Correlation between the unique dispersion features of a $\mathrm{PQC}$ and a zero-refractive index at the Dirac point is discussed later.

The eigenmodes in PQCs can either be localized or extended. For localized states, the energy is confined at a specific position and intensity decays exponentially away from the confinement center. For extended states, the fields spread throughout the sample so that energy can propagate outwards to the surrounding medium. Both modes in a finite-sized sample can be characterized by the normalized participation ratio defined as $P=$ $\left(\left(\int_{\text {cell }}\left|E_{z}\right|^{2} d \mathbf{r}\right)^{2} / \int_{\text {cell }}\left|E_{z}\right|^{4} d \mathbf{r}\right) / N$ [26], where $E_{z}$ is the maximum (or average) electric field around the rod, and $N$ is the number of rods in a PQC. If the intensity at each site is equal, $P=1$. It is found that $P \sim 1$ for the modes in the conical dispersion manifold for both the first level and second level supercells. For example, a mode with $f=$ $0.56 c / a$ belonging to the cone has $P=0.96$, presenting that it is extended (Supplemental Material [25], Appendix A).

It is interesting to see whether PQC with conical dispersions can be regarded as zero-refractive-index material. If it could, the extended state should have a constant phase throughout the entire PQC at the Dirac frequency. This is demonstrated for a finite-sized quasicrystal. For a 12-fold PQC, energy transport was measured by placing a source at the Dirac frequency of $0.5584 c / a$ and near the center of the quasicrystal with the first level crystalline approximant parameters. Results of the internal 
field distributions of the sample carrying 2203 rods and 4231 rods in Figs. 2(a) and 2(b), respectively, are essentially the same. The intensity and the phase are almost uniform in the entire quasicrystal, and the waves are strongly leaky outward. In addition, such a "zero phase change" feature persists no matter how the boundaries of quasicrystals are shaped as long as the conical dispersion persists (Supplemental Material [25], Appendix C). Such extended states with zero phase change in the 12-fold PQC with the bigger level crystalline approximants are illustrated in the Supplemental Material [25], Appendix C. As a control calculation, the sample is excited far away from the Dirac frequency $(f=0.448 c / a)$. Most of the source energy remains localized at several particular positions and trapped inside the quasicrystals [Figs. 2(c) and 2(d)], indicating that the excited modes are localized rather than extended.

To demonstrate that the PQC behaves like a zerorefractive-index medium, a prism is constructed using the 12-fold PQC. The length of two right-angle sides of the prism are $45.38 a$ and $26.2 a$, and the sample is constructed according to the first level crystalline approximant parameters. A transverse magnetic plane wave illuminates the bottom interface (as shown schematically by the black arrows in Fig. 3). The incident angle at the slant boundary of the prism is $\theta_{o}=60^{\circ}$. The refraction behavior at the boundary will be investigated near the Dirac frequency. By measuring the angle between the refracted wave fronts (black lines) and the inclined surface, the refraction angles
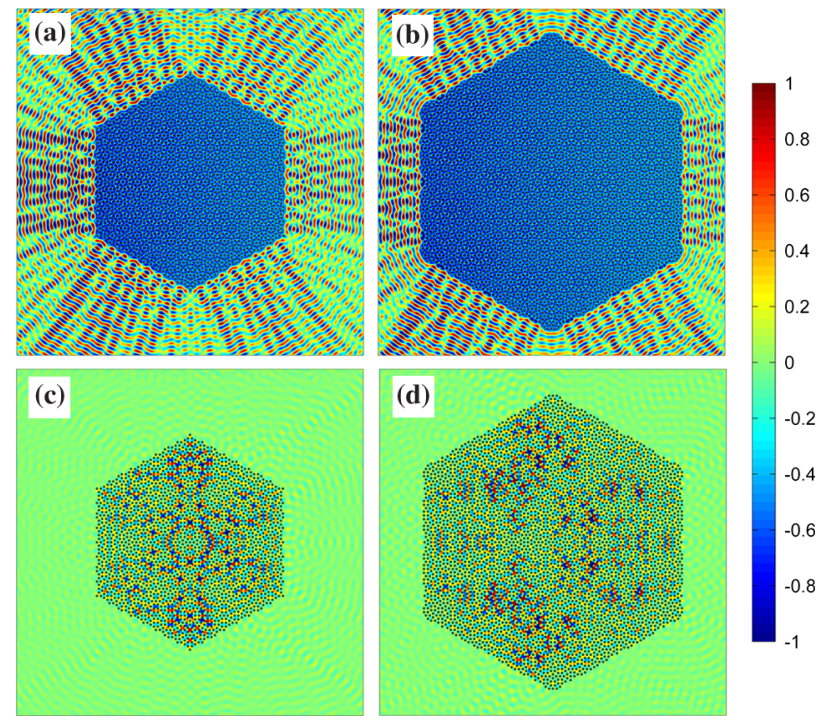

FIG. 2 (color online). Normalized electric fields in the 12-fold quasicrystals with different number of rods. (a) and (b) Extended states with constant phase excited at the Dirac frequency of $0.5584 c / a$. (c) and (d) Localized modes at the frequency of $0.448 c / a$. Zero phase extended states persist as the size becomes larger. The extended states allow energy to be transported while the localized modes trap energy inside the quasicrystal. The patterns are the real part of the fields. are found to be $\theta_{i}=-1.92^{\circ} / 0.06^{\circ} / 6.2^{\circ}$ at the frequencies of $0.554 c / a, 0.5584 c / a$, and $0.58 c / a$, respectively. Results indicate that the refractions change from negative to zero to positive as the frequency increases. The field patterns inside the sample also give information on the phase change. The color is nearly uniformly blue, as shown in Fig. 3(b), while it varies inside the prism, as shown in Figs. 3(a) and 3(c), a behavior that is consistent with the results from the effective medium, as shown in Appendix E of the Supplemental Material [25]. Furthermore, we introduce a term $\delta=\sin \theta_{i}-$ $n_{\text {eff }} \sin \theta_{o}$ to measure the validity of Snell's law, where $n_{\text {eff }}$ is the effective refractive index and is defined by measuring the wavelength within the quasicrystal (Supplemental Material [25], Appendix D). The red curve in Fig. 3(d) always tends to zero, confirming that Snell's law is valid from $0.545 c / a$ to $0.6 c / a$. Figure 3(d) also illustrates that the effective refractive index varies almost linearly with the working frequency and is close to zero within the interesting frequency.

It is well-known that a zero-index medium can "cloak" an object inside a channel [27-29]. To experimentally demonstrate it, a channel filled with the zero-index 12-fold PQCs is fabricated by alumina rods with $\varepsilon=8.8$ and $r=3.75 \mathrm{~mm}$ [Fig. 4(a)]. The nearest-neighbor distance between rods is $a=17.37 \mathrm{~mm}$ and the Dirac frequency is $10.42 \mathrm{GHz}$. The rods are placed between two perfect electric conductor (PEC) plates at a distance less than half a wavelength. At such a distance, the waveguide system can be treated accurately using $2 \mathrm{D}$ calculations as the waveguide only allows zero-order single-mode propagation. As it is impossible to terminate the quasicrystal with a flat boundary, the boundary modification is needed to preserve the straight wave front for the incident and exit waves [30]. The obstacle is a metal rod (blue) with a radius of $47.5 \mathrm{~mm}$ located at the center of the channel.

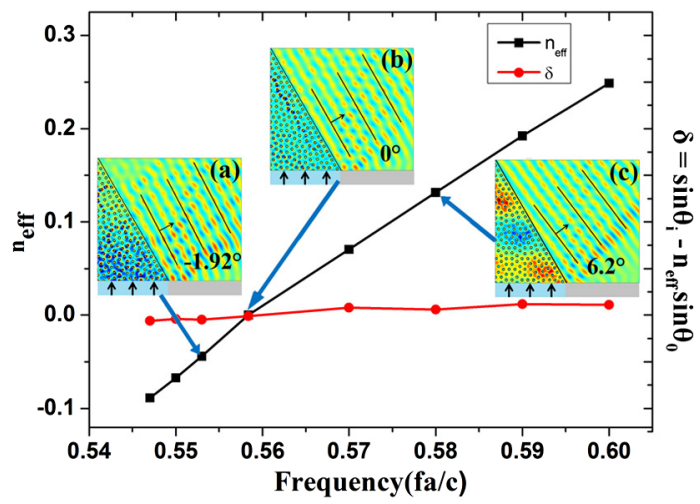

FIG. 3 (color online). Demonstration of zero refraction and Snell's law. (a)-(c) Field patterns at three different frequencies. The plane wave is incident to the right-angle prism (black arrows) and forms the wave front (thin black lines) traveling upper right. (d) The effective refractive index (black) and the term $\delta=$ $\sin \theta_{i}-n_{\text {eff }} \sin \theta_{o}$ (red) as a function of frequency. The term $\delta$ being approximate to zero shows that light refraction is governed by Snell's law. 
(a)

Quasicrystal with 12-fold symmetry

(b)Cloaking effect in near-zero quasicrystal
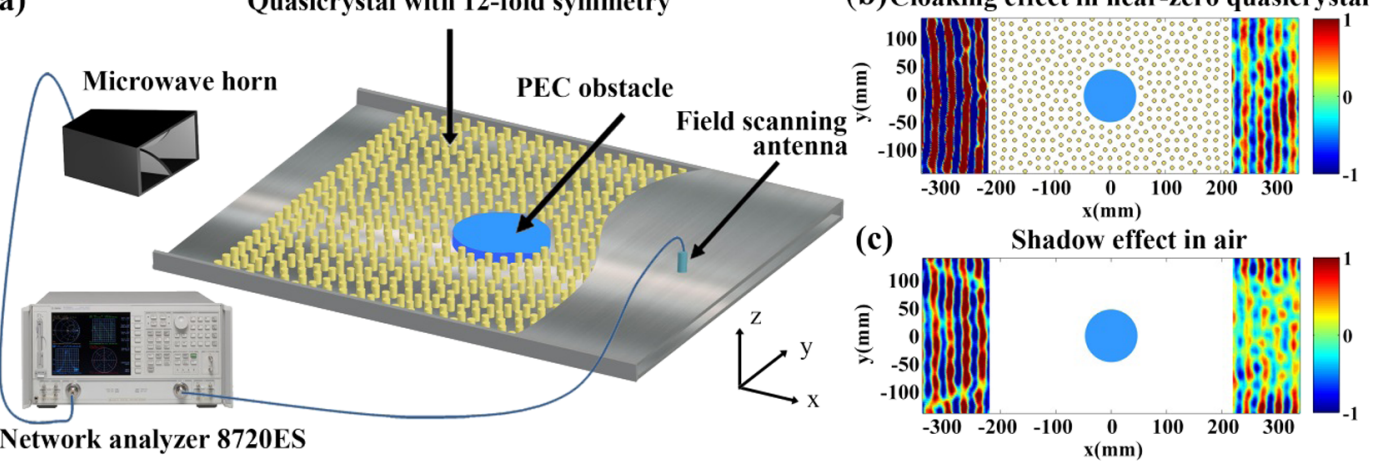

FIG. 4 (color online). (a) Schematic of the experimental setup for field measurement. The sample is made by alumina rods (yellow) arranged in a 12-fold quasicrystalline array, sandwiched between two aluminum plates. A PEC obstacle (blue) is placed in the channel. (b) Measured $E_{z}$ fields with quasicrystalline array at $10.542 \mathrm{GHz}$, and the wave front retrieval on the exit side. (c) Control experiment of an obstacle in the channel without quasicrystalline array. Note the shadow cast by the obstacle on the exit side and the interference due to reflection on the left side. All the fields are normalized.

The boundary walls of the channel are artificial perfect magnetic conductors (high impedance surface) and the $E_{z}$ source is placed $150 \mathrm{~cm}$ away from the left channel port to achieve plane wave fronts. The areas of the field measurements on both sides are $30 \mathrm{~mm} \times 20 \mathrm{~mm}$ as measured by Network Analyzer 8720ES from 10.4 to $10.6 \mathrm{GHz}$. From the recovery of the transmitted fields and the preservation of the plane wave front at $10.542 \mathrm{GHz}$, as shown in Fig. 4(b), it can be reasoned that the metal rods were not there. Further, numerical results show that there is almost no phase change at $10.542 \mathrm{GHz}$ as $n_{\text {eff }}=0.047$. (Complete results for fields both outside and inside the quasicrystal at different frequencies are summarized in Appendix F of the Supplemental Material [25].) Phase change near the exit boundary (at $x$ approximately $210 \mathrm{~mm}$ ) is almost the same in experiments and simulations. This gives evidence to the near-zero index properties near the Dirac frequency region. When the quasicrystal sample is removed, a shadow area behind the metal rod on the right-side area is observed. At the same time, interference tracks clearly appear on the left side due to backscattering of the obstacle [Fig. 4(c)].

Zero refractive index can be used to realize asymmetric transmission if the left and right boundaries of the structure have broken spatial symmetry [31]. A simple symmetrybreaking prism-shaped object is shown in Fig. 5. When the right-going plane wave is incident normal to the boundary of zero-index PQC, high transmittance can be achieved. However, the left-going plane wave suffers strong reflection given a $0^{\circ}$ critical incident angle from the air to zeroindex PQC. Such asymmetric transport is demonstrated by simulation results in Figs. 5(a) and 5(b). It is induced by an inversion-symmetry breaking scatterer inside a multichannel waveguide, rather than nonreciprocity. Furthermore, the transmission ratio of the right-going and the left-going waves is significantly enhanced. To demonstrate the phenomenon experimentally, the $E_{z}$ field is measured at a given $x$ line and the calculated transmission ratio by the definition of $\int\left|E_{z}^{\text {right }}\right|^{2} d y / \int\left|E_{z}^{\text {left }}\right|^{2} d y$, where
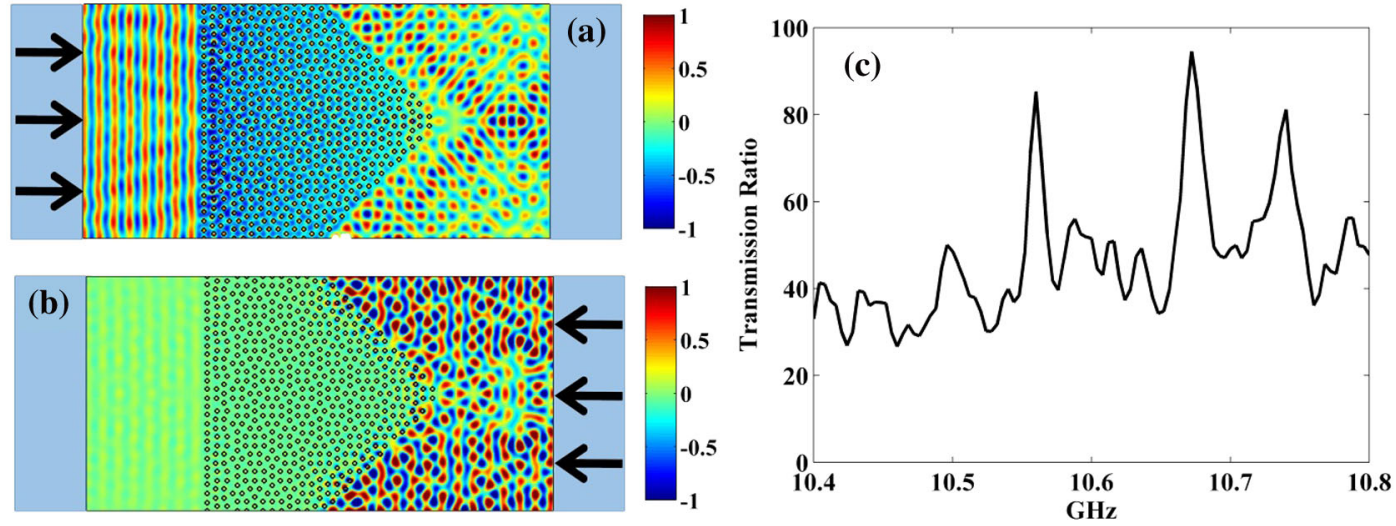

FIG. 5 (color online). (a) and (b) Normalized calculated field pattern in a multichannel waveguide containing a quasicrystalline prism, showing asymmetric transmission at the Dirac frequency of $10.42 \mathrm{GHz}$. (a) The left-going waves have high transmittance while (b) the right-going waves suffer from nearly total reflection. (c) Measured transmission ratio between the left and right incidence wave. The ratio is above 26 and can reach the maximum of 94 at the frequency of $10.67 \mathrm{GHz}$. 
$\left|E_{z}^{\text {right }}\right|$ and $\left|E_{z}^{\text {left }}\right|$ are the amplitude of output electric fields for the right- and left-going waves. It is found that the ratio is always above 26 from 10.4 to $10.6 \mathrm{GHz}$ and can reach 94 times at a certain frequency [Fig. 5(c)].

Effective zero-refractive index has been long associated with conical dispersions at $\mathbf{k}=\mathbf{0}$ [1] within the framework of a periodic structure. This Letter shows that conical dispersions and effective zero-refractive index can go beyond periodicity. The essence of the physics is that conical dispersions require high symmetry to ensure linear bands crossing each other without level repulsion, and quasicrystals provide high enough rotation symmetry to support the cones. We show feasibility in some quasicrystals but the requirements are stringent. Difficulties include finding cones in fivefold Penrose tiled quasicrystals. Thus, exhibiting zero-index behavior is more demanding than exhibiting photonic band gaps, as we know that quasicrystals of all symmetries support band gaps $[12,13]$. Even amorphous photonic crystals support band gaps [32], but disorder as a precursor to level repulsion can eliminate the conical dispersion (Supplemental Material [25], Appendix G). This is unlike using quasicrystals to realize the effective negative index in which the negative index will persist independent of the detailed arrangement of the "atoms" as long as the filling ratio is correct [16]. In addition, one can also extend the concepts to optical frequencies as the PQC is constructed by all-dielectric and low-loss rods. The conical dispersion and effective zero-index behaviors can be observed by changing the filling ratio of the quasicrystal, with the same procedures discussed above.

In summary, it was shown that some PQCs could possess conical dispersions at $\mathbf{k}=\mathbf{0}$. The modes in the conical dispersion are extended states with almost constant phase. Experimental evidence suggesting a zero refractive index includes refraction angle measurements, verification of constant phase for modes near the Dirac frequency, cloaking of scattering objects inside a waveguide, and asymmetric transmission through prism-shaped objects inside a multichannel waveguide.

This work is supported by Grants of NSFC (No. 11274396), 973 project (No. 2014CB931701), HKRGC (AOE/P-02/12), and IRT13042. J. W. D. is also supported by Guangdong Natural Science Funds for Distinguished Young Scholar (S2013050015694). J. W. D. is visiting in University of California, Berkeley.

*dongjwen@mail.sysu.edu.cn

[1] X. Huang, Y. Lai, Z. H. Hang, H. Zheng, and C. T. Chan, Nat. Mater. 10, 582 (2011).

[2] C. T. Chan, Z. H. Hang, and X. Huang, Adv. OptoElectron. 2012, 313984 (2012).

[3] C. T. Chan, X. Huang, F. Liu, and Z. H. Hang, PIERS B 44, 163 (2012).

[4] K. Sakoda, Opt. Express 20, 3898 (2012).

[5] K. Sakoda, Opt. Express 20, 25181 (2012).
[6] J. Mei, Y. Wu, C. T. Chan, and Z. Q. Zhang, Phys. Rev. B 86, 035141 (2012).

[7] J. Luo and Yun Lai, Sci. China Inform. Sci. 56, 1 (2013).

[8] J. Schilling, Nat. Photonics 5, 449 (2011).

[9] H. Iizuka and N. Engheta, Phys. Rev. B 90, 115412 (2014).

[10] P. Moitra, Y. Yang, Z. Anderson, I. I. Kravchenko, D. P. Briggs, and J. Valentine, Nat. Photonics 7, 791 (2013).

[11] Y. Wu, J. Li, Z.-Q. Zhang, and C. T. Chan, Phys. Rev. B 74, 085111 (2006).

[12] M. E. Zoorob, M. D. B. Charlton, G. J. Parker, J. J. Baumberg, and M. C. Netti, Nature (London) 404, 740 (2000).

[13] Y. S. Chan, C. T. Chan, and Z. Y. Liu, Phys. Rev. Lett. 80, 956 (1998).

[14] M. Bayindir, E. Cubukcu, I. Bulu, and E. Ozbay, Phys. Rev. B 63, 161104(R) (2001).

[15] M. Florescu, S. Torquato, and P. J. Steinhardt, Phys. Rev. B 80, 155112 (2009).

[16] Z. Feng, X. Zhang, Y. Wang, Z.-Y. Li, B. Cheng, and D.-Z. Zhang, Phys. Rev. Lett. 94, 247402 (2005).

[17] M. Notomi, H. Suzuki, T. Tamamura, and K. Edagawa, Phys. Rev. Lett. 92, 123906 (2004).

[18] K. Nozaki and T. Baba, Appl. Phys. Lett. 84, 4875 (2004).

[19] S.-K. Kim, J.-H. Lee, S.-H. Kim, I.-K. Hwang, Y.-H. Lee, and S.-B. Kim, Appl. Phys. Lett. 86, 031101 (2005).

[20] R. T. Bratfalean, A. C. Peacock, N. G. R. Broderick, K. Gallo, and R. Lewen, Opt. Lett. 30, 424(2005).

[21] R. Lifshitz, A. Arie, and A. Bahabad, Phys. Rev. Lett. 95, 133901 (2005).

[22] C. A. B. Clausen, Y.S. Kivshar, O. Bang, and P. L. Christiansen, Phys. Rev. Lett. 83, 4740 (1999).

[23] S. Fischer, A. Exnera, K. Zielskea, J. Perlichb, S. Deloudic, W. Steurerc, P. Lindnerd, and S. Förstere, Proc. Natl. Acad. Sci. U.S.A. 108, 1810 (2011).

[24] M. Oxborrow and C. L. Henley, Phys. Rev. B 48, 6966 (1993).

[25] See Supplemental Material at http://link.aps.org/ supplemental/10.1103/PhysRevLett.114.163901 for the conical dispersion and eigenmodes analysis of both 12-and 8-fold PQCs, and effective medium simulation and the disorder evaluation.

[26] J.-W. Dong, K. H. Fung, C. T. Chan, and H.-Z. Wang, Phys. Rev. B 80, 155118 (2009).

[27] A. Alu, M. G. Silveirinha, A. Salandrino, and N. Engheta, Phys. Rev. B 75, 155410 (2007).

[28] J. Hao, W. Yan, and M. Qiu, Appl. Phys. Lett. 96, 101109 (2010).

[29] M. Silveirinha and N. Engheta, Phys. Rev. Lett. 97, 157403 (2006).

[30] For boundary modifications, the rods in the first leftmost (rightmost) column are fixed at the value of $x_{1}^{\prime}$. The rods in the first four columns need to be modified to $x_{i}^{\prime}=\alpha_{i} x_{1}^{\prime}+\left(1-\alpha_{i}\right) x_{4}$. Here, $\alpha_{i}=1,0.66,0.33,0$ for $i=1,2,3,4$ and $x_{4}$ is the original coordinate of the rods in the fourth column. After such modification, the characteristics of the incident and exit plane waves can be preserved.

[31] Y. Fu, L. Xu, Z. H. Hang, and H. Chen, Appl. Phys. Lett. 104, 193509 (2014).

[32] K. Edagawa, S. Kanoko, and M. Notomi, Phys. Rev. Lett. 100, 013901 (2008). 